\title{
Prévision de la digestibilité des graminées, des trèfles et des associations graminées-trèfle blanc à partir de leur composition chimique et de la digestibilité par la cellulase
}

\author{
R Giovanni ${ }^{1}$, J Scéhovic ${ }^{2}$, JL Peyraud ${ }^{1}$, J Aufrère ${ }^{3}$ \\ 1INRA, station de recherches sur la vache laitière, St-Gilles, 35590 L'Hermitage, France; \\ 2Station fédérale de recherche agronomique Changins, 1260 Nyon, Suisse; \\ 3INRA - CRZV Theix, station de recherches sur la nutrition des herbivores, \\ 63122 Saint-Genès-Champanelle, France
}

\begin{abstract}
Summary - Prediction of organic matter digestibility of grass, clover and grass-clover mixtures from the chemical composition and cellulase digestibility of the forage. Chemical constituents of grasses are not able to predict organic matter digestibility (DMO) with precision $(S r>2.0)$, although insoluble phenolic compounds (CPFI) and silica improve the prediction model $(S r=1.6)$. Lignin alone represents a secure parameter to estimate white and red clover organic matter digestibility. However, DM digestibility by the cellulase method appears to be the most pertinent parameter for all green forage, mixture swards included $(S r=1.8)$.
\end{abstract}

Le but de cette étude a été de rechercher les liaisons existant entre la digestibilité in vivo de la matière organique (DMO) des fourrages verts de graminées, de trèfles et de leur association, la digestibilité "Cellulase» de leur matière sèche et leur composition chimique.

Matériel et méthodes - Les prairies de graminées (ray-grass anglais, RGA; dactyle, DAC; brômes, BRO), de légumineuses (trèfles blanc et violet) et les associations RGA/TB et $\mathrm{DAC} / \mathrm{TB}$, ont été exploitées du stade "feuillu" au stade "début épiaison" pour le premier aycle et à l'âge de 4-6 semaines pour les repousses du second cycle. Les mesures de digestibilité de l'herbe coupée chaque jour ont été effectuées sur 6 moutons mâles castrés standard, maintenus en cage et alimentés à volonté (15\% de refus), sauf avec le trèfle blanc pur $(65 \mathrm{~g} \mathrm{MS} / \mathrm{kg}$ $\left.\mathrm{p}^{0.75}\right)$. Chaque période de mesures a été de $5 \mathrm{j}$. Un échantillon des fourrages prélevé journellement a été séché $\left(55^{\circ}-3\right.$ j) et conservé à l'abri de la lumière pour le dosage, selon les méthodes proposées par Scéhovic (1979), du résidu au détergent neutre (matières fibreuses totales, MFT \% MS), du résidu au détergent acide (lignocellulose, LC) et de ses sous-fractions dénommées cellulose vraie (CV) et lignine (LI), ainsi que de la silice (SI), des composés phénoliques solubles (CPFS) et insolubles (CPFI). La digestibilité "cellulase" de la matière sèche ( $D$ cellulase) a été réalisée par la méthode d'Aufrère et Michalet-Doreau (1988).

Résultats et discussion - Pour les graminées pures, l'utilisation des paramètres chimiques précédents (exprimés en $\% \mathrm{~ms}$ ) pris isolément, y compris la teneur en MAT, conduit à une précision insuffisante (tableau I). L'utilisation conjointe des teneurs en MAT, MFT ou CPFI apporte une amélioration sensible de la précision encore renforcée par SI. Cependant, it demeure un effet "espèce " important $(S r=1,59$ et 2,10 avec et sans l'effet "espèce") :

$$
\begin{gathered}
\text { DMO }(\%)=74,2+2,88 \mathrm{MAT}-0,081 \\
\text { MAT }^{2}-1,68 \mathrm{SI}-14,48 \mathrm{CPFI}+\Delta \\
\left(\begin{array}{r}
\Delta=+1,6 \mathrm{RGA},+0,6 \mathrm{DAC},-2,2 \mathrm{BRO}) \\
n=33 \quad r=0,94 \quad \mathrm{~S} r=1,59
\end{array}\right.
\end{gathered}
$$


Cette précision est voisine de celle permise par D cellulase (en \%), sauf pour l'effet " espèce " $(S r=1,64$ et 1,87 avec et sans effet "espèce") :

DMO $(\%)=43,87+0,461$ D cellulase $+\Delta$ $(\Delta=+1,4 \mathrm{RGA},-1,6 \mathrm{DAC},+0,2 \mathrm{BRO})$

$$
n=33 \quad r=0,92 \quad S r=1,64
$$

Pour les trèfles, la lignine seule est un paramètre très satisfaisant de la prévision de la DMO, sensiblement meilleur que la $D$ cellulase et aucun autre paramètre chimique ne vient l'améliorer :

$$
\begin{aligned}
& \operatorname{DMO}(\%)= 90,56-1,792 \mathrm{LI} \\
& n=24 \quad r=0,88 \quad S r=1,44
\end{aligned}
$$

Pour les associations graminées-trèfle blanc, la prévision de la DMO est peu précise, quel que soit le paramètre chim que utilisé $(S r>2,2)$. Elle est un peu meilleure à partir de $D$ cellulase : DMO $(\%)=38,8+0,507 \mathrm{D}$ cellulase

$$
n=15 \quad r=0,77 \quad S r=2,05
$$

En outre, la $D$ cellulase permet de prévoir de façon assez satisfaisante la DMO de tous les fourrages étudiés:

$$
\begin{gathered}
\text { DMO }(\%)=37,5+0,543 \text { D cellulase }+\Delta \\
n=72 \quad r=0,87 \quad S r=1,83
\end{gathered}
$$

$(\Delta=+1,02$ graminées, $+0,32$ trèfles, $-1,33$ associations)

L'équation plus générale proposée par Aufrère et Michalet-Doreau (1988) conduit à une sous-estimation moyenne de 2,3 points (+2 à -7 pts) de la DMO des graminées en vert mesurée dans ces essais.

En conclusion, l'intérêt de $\mathrm{D}$ cellulase pour prévoir la DMO des fourrages verts est à nouveau confirmé. Toutefois, pour les graminées pures, les teneurs en CPFI peuvent apporter une précision supplémentaire par comparaison aux paramètres indicatifs des parois. Pour les trèfles, la lignine seule représente un paramètre de prédiction très pertinent.

\begin{tabular}{|c|c|c|c|c|c|c|}
\hline \multirow{3}{*}{$\begin{array}{l}\text { Paramètres } \\
\text { DMO (\%) } \\
\text { Corrélation - Écart type }\end{array}$} & \multirow{2}{*}{\multicolumn{2}{|c|}{$\frac{\begin{array}{c}\text { Graminées } \\
(\mathrm{n}=33)\end{array}}{68-84}$}} & \multirow{2}{*}{\multicolumn{2}{|c|}{$\begin{array}{c}\begin{array}{c}\text { Trèfles } \\
(\mathrm{n}=24)\end{array} \\
74-85\end{array}$}} & \multirow{2}{*}{\multicolumn{2}{|c|}{$\begin{array}{c}\begin{array}{c}\text { Associations } \\
(\mathrm{n}=15)\end{array} \\
71.83\end{array}$}} \\
\hline & & & & & & \\
\hline & $r$ & $S r$ & $r$ & $S r$ & $r$ & $S r$ \\
\hline $\begin{array}{l}\text { Matières fibreuses totales (MFT) } \\
\text { Lignocellulose (LC) } \\
\text { Cellulose vraie (CV) } \\
\text { Lignine (LI) } \\
\text { Composés phénoliques insol (CPFI) } \\
\text { Digestibilité MS cellulase }\end{array}$ & $\begin{array}{l}0,83 \\
0,79 \\
0,77 \\
0,81 \\
0,83 \\
0,92\end{array}$ & $\begin{array}{l}2,49 \\
2,71 \\
2,85 \\
2,59 \\
2,46 \\
1,64\end{array}$ & $\begin{array}{l}0,78 \\
0,86 \\
0,80 \\
0,88 \\
0,74 \\
0,82\end{array}$ & $\begin{array}{l}1,98 \\
1,59 \\
1,92 \\
1,44 \\
1,83 \\
1,73\end{array}$ & $\begin{array}{l}\text { NS } \\
0,51 \\
\text { NS } \\
0,54 \\
\text { NS } \\
0,77\end{array}$ & $\begin{array}{l}\text { NS } \\
2,71 \\
\text { NS } \\
2,66 \\
\text { NS } \\
2,05\end{array}$ \\
\hline $\begin{aligned} \mathrm{MAT}+\mathrm{MAT}^{2} & +\mathrm{CPFI} \\
& +\mathrm{CPFI}+\mathrm{Si} \\
& +\mathrm{CPFI}+\mathrm{LI}^{\mathrm{a}} \\
\mathrm{CPFI}+\mathrm{LI} & \end{aligned}$ & $\begin{array}{l}0,93 \\
0,94 \\
0,86\end{array}$ & $\begin{array}{l}1,71 \\
1,59 \\
2,21\end{array}$ & $\begin{array}{l}\text { NS } \\
\text { NS } \\
\text { NS } \\
0,67\end{array}$ & $\begin{array}{l}\text { NS } \\
\text { NS } \\
\text { NS } \\
2,44\end{array}$ & $\begin{array}{l}\text { NS } \\
\text { NS } \\
0,86 \\
0,87\end{array}$ & $\begin{array}{l}\text { NS } \\
\text { NS } \\
2,21 \\
2,44\end{array}$ \\
\hline
\end{tabular}

Scéhovic J (1979) Fourrages 57-78

Aufrère J, Michalet-Doreau $B(1988)$ Ann Feed Sci Technol 20, 203-218

Tableau 1. Coefficient de corrélation $(r)$ et écart type résiduel $(S r)$ de la prévision de la DMO à partir de la composition chimique des fourrages et de la digestibilité MS par la cellulase.

a Prévision établie pour un ensemble regroupant graminées pures et associations. 